\title{
Secado de maderas tropicales en estufas convencionales
}

\author{
David Zavala Zavala ${ }^{1}$
}

\begin{abstract}
RESUMEN
Algunos de los problemas en el secado de la madera de especies tropicales en estufas convencionales se deben a la diversidad de sus propiedades tecnológicas, a la dificultad de contar con volúmenes adecuados de una misma especie que permitan adaptar los procesos de secado (secuelas) acordes a las características de sus maderas, y al alto costo del proceso que determina la capacidad rentable de las estufas y limita su utilización para secar volúmenes pequeños. Una alternativa para contrarrestar las limitantes señaladas, es formar grupos de especies semejantes, para integrar volúmenes acordes con las capacidades de las estufas, para aplicarles las mismas secuelas de secado. El presente trabajo se realizó con la finalidad de integrar grupos de especies de maderas tropicales que se pudiesen secar con una misma secuela para hacer más práctico su secado en estufas convencionales. Para este estudio se utilizó la madera aserrada de 33 especies tropicales, que se integraron en dos grupos con base en su densidad básica, 16 especies con densidad básica de 0.30 a $0.60 \mathrm{gr} / \mathrm{cm}^{3}$, y 17 de 0.60 a 0.90 $\mathrm{gr} / \mathrm{cm}^{3}$. A cada grupo se le formuló una secuela de secado, que se evaluó en una estufa convencional, analizando los defectos de cada tabla, antes y después del secado (rajaduras, torceduras, acanalamiento, arqueamiento y encorvado). En general, las dos secuelas aplicadas a los
\end{abstract}

dos grupos de especies generaron resultados favorables al no presentarse defectos significativos debidos al proceso de secado. En el grupo de especies de densidad básica menor de $0.60 \mathrm{gr} / \mathrm{cm}^{3}, 10$ se secaron más rápido que las otras 6 , por lo que se podrían subagrupar para optimizar las estufas de secado aplicándoles otra secuela más rápida. Con la secuela utilizada para las especies con densidad básica mayor de $0.60 \mathrm{gr} / \mathrm{cm}^{3}, 7 \mathrm{de}$ ellas se secaron en aproximadamente la mitad del tiempo (10-14días) en relación a las otras 10 , siendo posible subagruparlas también para optimizar las estufas. De no ser factible reagrupar a las especies dentro de cada categoría, se sugiere que para lograr un proceso lo más uniforme posible, coincidan los contenidos de humedad y las dimensiones de la madera en cada uno de los dos grupos de especies analizados en este trabajo.

PALABRAS CLAVE:

Industria forestal, especies tropicales, secuelas de secado.

\footnotetext{
ABSTRACT

Some of the drying problems of tropical species in conventional kilns are associated with the diversity of their technological properties, with the difficulty of gathering adequate volumes of the same species to
}

1 Instituto Nacional de Investigaciones Forestales y Agropecuarias. SAGAR. Progreso 5. Del. Coyoacán. 04110 México, D.F. México.

Manuscrito recibido el 24 de febrero de 1999. Aceptado el 14 de Diciembre de 1999 
apply them a drying schedule according to the wood characteristics, and with the high cost of the process that determines the profitable capacity of the kilns and limits its utilization to dry small volumes of lumber. An alternative to overcome these limitations is to integrate groups of similar species in volumes according to the capacity of the kilns, to be able to apply them the same drying schedule. This study was performed with the goal of integrating groups of tropical wood species that could be dried with the same schedule in order to make feasible the drying process in conventional kilns. For this study, the sawn lumber of 33 tropical species was used, which was integrated in two groups according to their specific gravity, 16 species with specific gravity of 0.30 to 0.60 , and 17 with 0.60 to 0.90. A drying schedule was formulated for each group, that was evaluated in a conventional kiln, analyzing the deffects of each board before and after the drying process (checks, twist, cup, bow and crook). In general, the two schedules applied to the two groups of species were adequate since no significant defects due to the drying process were observed. In the group of species with specific gravity below $0.60,10$ of them dried faster than the other 6 , which suggest the possibility of subgrouping them to optimize the used of the dry kilns applying them a faster schedule. With the schedule for the species with specific gravity higher than $0.60,7$ of them dried at aproximately half of the time (10 - 14 days) than the other 10, which suggests the possibility of subgrouping them to optimize the use of dry kilns. If it is not possible to regroup the species within each category to achieve a uniform process, it is suggested to integrate the lumber with similar moisture content and dimensions in each one of the two groups analized in this study.

KEY WORDS:

Forest industries, tropical species, drying schedules.

\section{INTRODUCCION}

La madera que se obtiene a través del aserrío de las trozas, generalmente contiene una gran cantidad de agua que requiere eliminarse antes de que pueda ser procesada para la elaboración de la mayoría de los productos en los que se le utiliza, como muebles, puertas, parquet, lambrín, etc. Los procedimientos para eliminar el exceso de agua en la madera son muy variados, desde los que aprovechan las condiciones secantes del medio ambiente (secado al aire libre), las estufas de secado en sus diferentes modalidades, estufas convencionales, solares, dehumidificadores, de vacío, hasta los que utilizan micro-ondas $u$ ondas de alta frecuencia.

Se considera que la forma tradicional y más recomendable de realizar el secado de la madera en estufas, es formando cargas uniformes de una misma especie, con dimensiones y contenido de humedad similares. Sin embargo, en el caso de las maderas tropicales no siempre es factible adoptar este principio. Algunos de los problemas que se presentan con el secado en estufa de las maderas tropicales son el gran número de especies con características tecnológicas diferentes; la dificultad de contar con volúmenes adecuados de una misma especie, que permitan adaptar los procesos de secado (secuelas) a una madera específica; por otro lado, el alto costo del proceso que determina la capacidad rentable de las estufas limitando su utilización a volúmenes pequeños.

Una alternativa para contrarrestar las limitantes antes señaladas es formar grupos de especies con características tecnológicas semejantes, para integrar volúmenes acordes a las capacidades de las estufas, a los que se les puedan aplicar las mismas secuelas de secado. Algunas propiedades tecnológicas de la madera que pueden utilizarse como indicadores para agrupar estas especies son las 
características físico-anatómicas, como la densidad, el contenido de humedad, la permeabilidad, las contracciones (anisotropía), el tamaño y distribución de los vasos y la orientación del hilo. Las especies que integren a cada grupo se podrán secar bajo condiciones similares, esperándose también un comportamiento semejante en cuanto a la calidad del secado 0 al desarrollo de posibles defectos ocasionados por el proceso, como rajaduras, torceduras, acanalamiento y apanalamiento.

El presente trabajo se realizó con la finalidad de determinar secuelas de secado que se puedan aplicar a grupos de especies tropicales mexicanas, considerando la afinidad de algunas de sus características tecnológicas.

\section{ANTECEDENTES}

Se considera que la forma tradicional y más recomendable de realizar el secado de la madera en estufas, es formando cargas de una misma especie con dimensiones y contenido de humedad similares. Siguiendo esta práctica y con base en los trabajos de Bramhall (1975); Bramhall y Wellwood (1976); Pratt (1974); Rasmussen (1968); Simpson (1991), en el laboratorio de secado de la madera del INIFAP, se determinaron con buenos resultados las secuelas para distintas especies tropicales mexicanas presentadas en la Tabla 1.

De las secuelas de secado desarrolladas en otros países para madera de especies tropicales, algunas coinciden con las que vegetan en México o bien con algunas de sus características tecnológicas del género de la especie (Tabla 2) (Boone et.al., 1988; Hildebrand, 1970).

Como se señaló anteriormente, la forma más recomendable de secar en estufa a las maderas tropicales, es formando grupos de especies con características afines. La imposibilidad de la adopción de este principio en México, se considera que puede influir en el limitado aprovechamiento de algunas especies tropicales, por la dificultad de completar los volúmenes requeridos para las capacidades de las estufas de secado comerciales (generalmente mayores de 20,000 pies tabla).

En otros países se han realizado trabajos agrupando especies para secarlas en forma conjunta. La selección o integración de los grupos se ha basado en algunas características tecnológicas de las maderas, considerando la densidad, el contenido de humedad, la permeabilidad, la anisotropía, la configuración del grano o hilo de la madera, la proporción de albura y duramen, el contenido de extractivos y el tamaño de los vasos y rayos (Simpson, 1989). Este autor desarrolló un modelo matemático para agrupar especies tropicales, con la finalidad de secarlas dentro de cierto rango de tiempo y con el mínimo de defectos. Para el desarrollo del modelo utilizó la densidad básica, el contenido de humedad $(\mathrm{CH})$ inicial y los tiempos de secado de cada especie, determinados previamente al inicio del secado.

En su reporte (Simpson, 1989) cita el trabajo realizado por Bello (1966), sobre el secado de 23 especies filipinas agrupadas en 3 clases, basándose en el grado de dificultad del secado, así como el trabajo de Casin et.al. (1980), sobre el secado de 60 especies filipinas integradas en 4 grupos, basándose en las características de secado de cada especie (densidad, orientación del grano y contracciones); cita también los trabajos realizados por Ma (1972), quien desarrolló 5 secuelas de tiempo para mezcla de 80 maderas de latifoliadas de Taiwan, considerando velocidad de secado, uniformidad del C.H. final, contracciones y defectos, también menciona los trabajos de Keenan y Tejeda (1984), quienes desarrollaron 3 secuelas de secado para un grupo de 105 especies sudamericanas, 
Tabla 1. Secuelas para especies tropicales desarrolladas en el INIFAP.

\begin{tabular}{|c|c|c|c|}
\hline NOMBRE CIENTÍFICO & NOMBRE COMÚN & ESPESOR & SECUELA \\
\hline Bombax ellipticum & Amapola cedreada & $4 / 4 "$ & Inglesa H \\
\hline Brosimum alicastrum & Ramón & $4 / 4 "$ & T7 - B3 \\
\hline Bucida buceras & Pukté & $4 / 4 "$ & Inglesa C \\
\hline Bursera simaruba & Chacáh & $4 / 4 "$ & Inglesa D \\
\hline Calophyllum brasiliense & Barí & $4 / 4 "$ & T3 - D4 \\
\hline Cedrela mexicana & Cedro rojo & $4 / 4 "$ & Inglesa F \\
\hline Cordia dodecandra & Siricote & $6 / 4 "$ & Inglesa C \\
\hline Cordia eleagnoides & Bocote & $6 / 4 "$ & Inglesa C \\
\hline Enterolobium cyclocarpum & Guanacaste & $4 / 4 "$ & T8 - F4 \\
\hline Lonchocarpus castilloi & Machiche & $6 / 4 "$ & Inglesa G \\
\hline Manilkara zapota & Chicozapote & $4 / 4 "$ & Inglesa E \\
\hline Metopium brownei & Chechem negro & $4 / 4 "$ & Inglesa C \\
\hline Metopium brownei & Chechem negro & $4 / 4 "$ & T3 - D3 \\
\hline Piscidia communis & Jabín & $4 / 4 "$ & Inglesa E \\
\hline Platymiscium yucatanum & Granadillo & $4 / 4 "$ & Inglesa C \\
\hline Schizolobium parahybum & Guanacaste & $4 / 4 "$ & T5 - F4 \\
\hline Simarouba glauca & Pasa'ak & $4 / 4 "$ & Inglesa D \\
\hline Simarouba glauca & Pasa'ak & $4 / 4 "$ & T9 - A3 \\
\hline Swietenia macrophylla & Caoba & $4 / 4 "$ & T8 - D5 \\
\hline Terminalia amazonia & Tepezúchil & $3 / 4 "$ & T2 - C3 \\
\hline
\end{tabular}

Tabla 2. Secuelas de especies tropicales coincidentes con especies mexicanas.

\begin{tabular}{|c|c|c|c|c|}
\hline NOMBRE CIENTÍFICO & NOMBRE COMÚN & \multicolumn{2}{|c|}{ ESPESOR } & SECUELA \\
\hline $\begin{array}{l}\text { Calophyllum brasiliense } \\
\text { Cedrela spp } \\
\text { Ceiba pentandra } \\
\text { Enterolobium cyclocarpum } \\
\text { Lonchocarpus castilloi } \\
\text { Lysiloma spp } \\
\text { Swietenia macrophylla } \\
\text { Tabebuia spp } \\
\text { Tectona grandis } \\
\text { Terminalia amazonia }\end{array}$ & $\begin{array}{l}\text { Santa María } \\
\text { Cedro } \\
\text { Ceiba } \\
\text { Guanacaste } \\
\text { Machiche } \\
\text { Tzalam } \\
\text { Caoba } \\
\text { Flor de mayo } \\
\text { Teca } \\
\text { Tepezúchil }\end{array}$ & $\begin{array}{l}4 / 4 \\
4 / 4 \\
4 / 4 \\
4 / 4 \\
4 / 4 \\
4 / 4\end{array}$ & $\begin{array}{l}\text { T2 - } \\
\text { T10 } \\
\text { T10 } \\
\text { T6 - } \\
\text { T8 - } \\
\text { T3 - } \\
\text { T6 - } \\
\text { T6 - } \\
\text { T3 - }\end{array}$ & 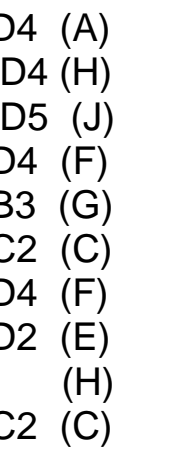 \\
\hline NOMBRE CIENTÍFICO & NOMBRE COMÚN & $\begin{array}{c}\text { DENSIDAD } \\
\text { BÁSICA } \\
\left(\mathrm{gr} / \mathrm{cm}^{3}\right)\end{array}$ & $\begin{array}{c}\text { CLAVE } \\
\text { SECUELA }\end{array}$ & $\begin{array}{l}\text { GRUPO } \\
\text { MADERA }\end{array}$ \\
\hline $\begin{array}{l}\text { Lonchocarpus castilloi } \\
\text { Cedrela mexicana } \\
\text { Ceiba pentandra } \\
\text { Calophyllum brasiliense } \\
\text { Swietenia macrophylla }\end{array}$ & $\begin{array}{l}\text { Machiche } \\
\text { Cedro rojo } \\
\text { Ceiba } \\
\text { Santa María } \\
\text { Caoba }\end{array}$ & $\begin{array}{l}0.90 \\
0.44 \\
0.26 \\
0.56 \\
0.49\end{array}$ & $\begin{array}{c}7 \\
7 \\
9-10 \\
2-3 \\
5\end{array}$ & $\begin{array}{l}4 \\
3 \\
7 \\
6 \\
3\end{array}$ \\
\hline
\end{tabular}


basados en el comportamiento de la madera expuesta al aire libre, para posteriormente derivar las secuelas de secado en estufa, con base en el tipo y severidad de los defectos de la madera.

\section{MATERIALES Y METODOLOGÍA}

Para la realización de este trabajo, se utilizó la madera de 33 especies que se integraron en dos grupos, los cuales se formaron con base en las densidades de las especies: el grupo uno con densidad básica de 0.30 a $0.60 \mathrm{gr} / \mathrm{cm}^{3}$ y el grupo dos con densidad básica mayor de $0.60 \mathrm{gr} / \mathrm{cm}^{3}$ (Tablas 3 y 4) (Torelli, 1983). De cada una de las especies indicadas, se utilizó una muestra de 8 tablas por carga de secado, de $2.50 \mathrm{~m}$ de largo, por anchos de 15 a 25 $\mathrm{cm}$ y espesor de $2.54 \mathrm{~cm}$.

Para evaluar el efecto de las secuelas de secado en las especies seleccionadas, se tomó como base la posible pérdida de volumen de madera y la calidad de cada tabla utilizada, por medio de su clasificación considerando las "Unidades de Corte", con base en el Proyecto de Norma para Clasificar Madera Aserrada de Latifoliadas (DDI, 1991). Para la evaluación y cuantificación de los defectos de las rajaduras o grietas, torceduras, arqueamiento, acanalamiento y encorvado de las tablas, se utilizó la Norma Oficial Mexicana NOM-EE-95-1980 (DGN, 1980). La cuantificación de cada uno de estos defectos se expresó en porcentaje de la desviación respecto de la dimensión total de la tabla donde se midió cada defecto, p.e. el acanalamiento se midió en el ancho de la tabla y el valor de la deformación se expresó como un porcentaje del ancho total de la tabla evaluada. Estos parámetros se determinaron en cada tabla seleccionada antes de iniciar el proceso de secado y después de que se concluyó. Por la diferencia de los parámetros indicados, se determinó el efecto de las secuelas de secado en cada una de las especies estudiadas.
El contenido de humedad inicial y final de la madera de cada tabla utilizada en el estudio, se determinó por medio de secciones de humedad que se obtuvieron de cada tabla al final del proceso de secado, registrando el peso inicial de las tablas completas antes de iniciar el secado y su peso al final del proceso, utilizando las siguientes relaciones (Rasmussen, 1968):

$$
\begin{aligned}
\% \mathrm{CH} & =\frac{\mathrm{Pi}-\mathrm{Pf}}{\mathrm{Pf}} * 100 \\
\mathrm{PSCm} & =\frac{\mathrm{Pm}(100)}{10} \\
\% \mathrm{CHm} & =\frac{\mathrm{POm}-\mathrm{PSCm}}{\mathrm{PSCm}} * 100
\end{aligned}
$$

Donde:

$\% \mathrm{CHs}=$ Contenido de humedad de la sección

$\mathrm{Pi}=$ Peso inicial de la sección, gr

$\mathrm{Pf}=$ Peso final de la sección, gr

$\mathrm{PSCm}=$ Peso seco calculado de la muestra al final del secado, gr

$\mathrm{Pm}=$ Peso de la muestra, gr

$\% \mathrm{CHm}=$ Contenido de humedad de la muestra

POm = Peso original de la muestra, gr

Para la determinación de los pesos de las tablas se utilizó una báscula con capacidad de $25 \mathrm{~kg}$ y con aproximación a gramos, para la determinación de los pesos de las secciones se utilizó una balanza con aproximación a décimas de gramo. Las secciones de humedad se deshidrataron en un horno con circulación forzada del aire a una temperatura de $100^{\circ} \mathrm{C}$. hasta lograr un peso constante.

Para la realización de los ensayos de secado se utilizó una estufa convencional experimental con capacidad de 500 pies tabla (pt), en la cual se controlaron, al igual que en las estufas de capacidad comercial, la temperatura, la humedad 
Tabla 3. Especies tropicales de densidad básica de 0.30 a $0.60 \mathrm{gr} / \mathrm{cm}^{3}$

\begin{tabular}{|c|c|c|}
\hline NOMBRE CIENTÍFICO & NOMBRE COMÚN & $\begin{array}{c}\text { DENSIDAD } \\
\mathrm{gr} / \mathrm{cm}^{3}\end{array}$ \\
\hline Schizolobium parahybum & Palo de picho & 0.30 \\
\hline Dendropanax arboreus & Sac-chacáh & 0.40 \\
\hline Poulsenia armata & Masamorro & 0.40 \\
\hline Swietenia macrophylla & Caoba & 0.42 \\
\hline Guatteria anomala & Zopo & 0.43 \\
\hline Pseudobombax ellipticum & Amapola & 0.44 \\
\hline Pterocarpus hayesii & Palo de sangre & 0.45 \\
\hline Spondias mombin & Jobo & 0.45 \\
\hline Nectandra sp. & Laurel & 0.46 \\
\hline Simarouba glauca & Pasa'ak & 0.46 \\
\hline Vochysia hondurensis & Maca blanca & 0.46 \\
\hline Talauma mexicana & Jolmashté & 0.49 \\
\hline Pachira aquatica & Palo de agua & 0.50 \\
\hline Pithecellobium leucocalyx & Guacibán & 0.52 \\
\hline Guarea glabra & Cedrillo & 0.56 \\
\hline Blepharidium mexicanum & Popiste & 0.60 \\
\hline
\end{tabular}

Tabla 4. Relación de especies tropicales con densidad básica alta

\begin{tabular}{|l|l|c||}
\hline \multicolumn{1}{|c|}{ NOMBRE CIENTÍFICO } & NOMBRE COMÚN & $\begin{array}{c}\text { DENSIDAD } \\
\mathrm{gr} / \mathrm{cm}^{3}\end{array}$ \\
\hline Misanteca pekii & Pimientillo & 0.60 \\
Zuelania guidonia & Trementino & 0.61 \\
Sickingia salvadorensis & Chacahuanté & 0.66 \\
Terminalia amazonia & Canshán & 0.66 \\
Vatairea lundellii & Tinco & 0.66 \\
Platimiscium yucatanum & Granadillo & 0.67 \\
Vitex gaumeri & Ya'axnik & 0.67 \\
Aspidosperma megalocarpon & Bayo & 0.67 \\
Ampelocera hottlei & Luín & 0.69 \\
Quercus anglohondurensis & Chiquinib & 0.69 \\
Brosimum alicastrum & Ramón & 0.73 \\
Lonchocarpus castilloi & Machiche & 0.74 \\
Dialium guianense & Guapaque & 0.80 \\
Dipholis stevensonii & Guaité & 0.80 \\
Sweetia panamensis & Chakté & 0.80 \\
Swartzia cubensis & Corazón azul & 0.83 \\
Manilkara zapota & Chicozapote & 0.90 \\
\hline \hline
\end{tabular}


relativa y la velocidad del aire. Las tablas se apilaron dentro de la estufa utilizando separadores o fajillas de $30 \times 5 \times 1.9 \mathrm{~cm}$ (4' $\times 2$ a $3 / 4$ plg) colocados a una distancia de $60 \mathrm{~cm} \mathrm{(2')} \mathrm{cada} \mathrm{uno.} \mathrm{En} \mathrm{la} \mathrm{"cama"} \mathrm{superior}$ de la carga de secado, se colocó una tara o peso muerto que sirvió para evitar el alabeo de las tablas de las últimas "camas".

A través del análisis de las secuelas del laboratorio de secado de la madera del INIFAP, aplicadas a especies tropicales y de las publicadas en la literatura, se seleccionaron las secuelas que se consideraron más adecuadas para las especies de este estudio. De este análisis se derivaron dos secuelas, una para maderas de densidad baja y media (Tabla 5) y la otra para las de densidad alta (Tabla $6)$, las cuales se aplicaron a los dos grupos de especies que se integraron en este trabajo.

Para el control del proceso de secado y para la determinación de los cambios de etapa de las secuelas, se utilizaron dos muestras por especie, con una dimensión de $120 \mathrm{~cm} \mathrm{(4')} \mathrm{de} \mathrm{longitud,} \mathrm{las} \mathrm{cuales} \mathrm{se}$ obtuvieron de la parte media de las tablas. De los extremos de estas muestras se generaron dos secciones de humedad de 1 plg de ancho que se utilizaron para determinar el contenido de humedad de las muestras y con estos, el peso seco calculado de cada muestra, por medio del procedimiento y las fórmulas descritas anteriormente. Las muestras se pesaron y evaluaron diariamente para determinarles su contenido de humedad actual y el posible desarrollo de defectos causados por el secado. Se determinó el contenido de humedad diario de las muestras para definir los cambios de etapa de la secuela utilizando la relación:

$$
\% \mathrm{CHa}=\frac{\mathrm{PAm}-\mathrm{PSCm}}{\mathrm{PSCm}} * 100
$$

Donde:

$\% \mathrm{CHa}=$ Contenido de humedad actual de la muestra
PAm = Peso diario de la muestra al momento de pesarla durante el proceso de secado, gr
PSCm = Peso seco calculado de la muestra, gr .

El control del proceso de secado se realizó con las muestras más húmedas, efectuando los cambios de etapa de la secuela de acuerdo al contenido de humedad de estas muestras. Se considera que si se hubiese tomado como referencia el contenido de humedad de las muestras más secas para los cambios de etapa de la secuela, seguramente se hubiera degradado la madera más húmeda.

Al final del proceso de secado, cada una de las tablas que integró la carga de madera, se evaluó nuevamente determinándole su contenido de humedad por el procedimiento descrito y se cuantificaron los posibles defectos desarrollados durante el secado (rajaduras, acanalamiento, arqueamiento, encorvado y torceduras).

A través del análisis de las características de cada una de las tablas, antes y después del secado, se determinó la eficiencia de la secuela en cada uno de los dos grupos de especies, con base en la evaluación de los defectos indicados.

\section{ANÁLISIS DE RESULTADOS}

De los ensayos realizados, con base en la evaluación de las características de los defectos de las tablas, del tiempo de secado y de los contenidos de humedad de la madera, antes y después de que se terminó el proceso; se considera que la secuela de secado para el grupo de maderas blandas, con densidad básica inferior a $0.60 \mathrm{gr} / \mathrm{cm}^{3}$ (Tabla 5), fue favorable por no afectar la calidad de las tablas y requerir tiempos de secado que se pueden considerar razonables para maderas blandas (entre 5 a 7 días). 
Tabla 5. Secuela de secado para mezcla de especies tropicales de densidad baja y media.

\begin{tabular}{||c|c|c|c|c|c||}
\hline $\begin{array}{c}\text { CONTENIDO } \\
\text { DE HUMEDAD }\end{array}$ & $\begin{array}{c}\text { TEMPERATURA } \\
\text { BULBO } \\
\text { SECO } \\
(\%)\end{array}$ & $\begin{array}{c}\text { DEPRESIÓN } \\
\left.{ }^{0} \mathrm{~F}\right)\end{array}$ & $\begin{array}{c}\text { TEMPERATURA } \\
\text { BULBO } \\
\text { HÚMEDO } \\
\left({ }^{0} \mathrm{~F}\right)\end{array}$ & $\begin{array}{c}\text { HUMEDAD } \\
\text { RELATIVA }\end{array}$ & $\begin{array}{c}\text { CONTENIDO } \\
\text { DE HUMEDAD } \\
\text { EQUILIBRIO } \\
(\%)\end{array}$ \\
\hline 35 & 130 & 9 & 121 & 76 & 13.0 \\
$30-30$ & 130 & 11 & 119 & 71 & 11.5 \\
$25-20$ & 140 & 15 & 125 & 64 & 9.5 \\
$20-15$ & 150 & 20 & 130 & 58 & 8.0 \\
$15-$ final & 160 & 35 & 125 & 43 & 6.0 \\
\hline
\end{tabular}

Tabla 6. Secuela de secado para mezcla de especies tropicales de densidad alta.

\begin{tabular}{|c|c|c|c|c|c|}
\hline $\begin{array}{c}\text { CONTENIDO } \\
\text { DE HUMEDAD } \\
(\%)\end{array}$ & $\begin{array}{c}\text { TEMPERATURA } \\
\text { BULBO } \\
\text { SECO } \\
\left({ }^{0} \mathrm{~F}\right)\end{array}$ & $\begin{array}{c}\text { DEPRESIÓN } \\
\left({ }^{0} \mathrm{~F}\right)\end{array}$ & $\begin{array}{c}\text { TEMPERATURA } \\
\text { BULBO } \\
\text { HÚMEDO } \\
\left({ }^{0} \mathrm{~F}\right)\end{array}$ & $\begin{array}{c}\text { HUMEDAD } \\
\text { RELATIVA } \\
(\%)\end{array}$ & $\begin{array}{c}\text { CONTENIDO } \\
\text { DE HUMEDAD } \\
\text { EQUILIBRIO } \\
(\%)\end{array}$ \\
\hline $\begin{array}{c}>35 \\
35-30 \\
30-25 \\
25-20 \\
20-15 \\
5-\text { final }\end{array}$ & $\begin{array}{l}110 \\
110 \\
115 \\
125 \\
140 \\
160\end{array}$ & $\begin{array}{r}3 \\
6 \\
10 \\
13 \\
18 \\
40\end{array}$ & $\begin{array}{l}107 \\
104 \\
105 \\
112 \\
122 \\
120\end{array}$ & $\begin{array}{l}90 \\
81 \\
71 \\
65 \\
58 \\
31\end{array}$ & $\begin{array}{r}19.0 \\
15.1 \\
12.1 \\
10.5 \\
8.7 \\
4.3\end{array}$ \\
\hline
\end{tabular}

Evaluación de la pérdida de humedad durante el secado en las especies de baja densidad. El rango del $\mathrm{CH}$ inicial entre especies fue muy amplio: de $48.5 \%$ en llora sangre y hasta $115.52 \%$ en maca blanca, lo cual no es lo más recomendable para cargas de madera para secarse en estufa, que es precisamente uno de los problemas del secado de las maderas tropicales (Tabla 7). Respecto al comportamiento de la madera durante el proceso de secado y su relación con la pérdida de humedad, de las especies estudiadas, la maca blanca, el palo de picho, el jobo, el pasa'ak y el cedrillo, tenian un $\mathrm{CH}$ mayor del $80 \%$. De estas especies, en la última etapa del secado el palo de picho, la maca blanca y el cedrillo presentaron también un $\mathrm{CH}$ más alto que el resto de las especies analizadas; para las otras dos, el jobo y el pasa'ak, el $\mathrm{CH}$ final fue más bajo, requiriendo un día menos de secado. Por otro lado, el sacchacáh, la caoba y el popiste, aún cuando su $\mathrm{CH}$ inicial fue relativamente más bajo que las cinco especies indicadas anteriormente, requirieron de un día más de secado que el resto de las especies listadas en la Tabla 7.

Si fuera posible integrar los volúmenes suficientes de madera para procesarse en estufas, se podrían formar dos grupos que se considera que se comportan en forma similar en el secado: masamorro, zopo, amapola, palo de sangre, jobo, laurel, pasa'ak, jolmashté, palo de agua y guacibán. A este grupo se les podría aplicar una secuela un poco más rápida que la analizada en este estudio. El otro grupo estaría integrado por palo de picho, sacchacáh, caoba, maca blanca, cedrillo y popiste. 
Tabla 7. Pérdida de humedad durante el secado en las maderas de baja densidad.

\begin{tabular}{|c|c|c|c|c|c|c|c|c|}
\hline \multirow{2}{*}{ ESPECIES } & \multicolumn{8}{|c|}{ TIEMPO DE SECADO EN DÍAS } \\
\hline & 0 & 1 & 2 & 3 & 4 & 5 & 6 & 7 \\
\hline & $(\%) \mathrm{CH}$ & $(\%) \mathrm{CH}$ & $(\%) \mathrm{CH}$ & $(\%) \mathrm{CH}$ & $(\%) \mathrm{CH}$ & $(\%) \mathrm{CH}$ & $(\%) \mathrm{CH}$ & $(\%) \mathrm{CH}$ \\
\hline Palo de picho & 98.92 & 68.2 & 42.8 & 33.6 & 27.2 & 16.9 & 10.57 & 8.94 \\
\hline Sac-chacáh & 56.21 & 40.7 & 27.1 & 21.2 & 16.5 & 10.5 & 9.14 & 7.71 \\
\hline Masamorro & 52.43 & 37.9 & 25.4 & 19.6 & 14.8 & 10.5 & 8.05 & \\
\hline Caoba & 53.38 & 38.2 & 26.9 & 20.5 & 15.2 & 11.0 & 9.33 & 8.15 \\
\hline Zopo & 57.98 & 40.3 & 26.9 & 22.5 & 15.9 & 10.6 & 7.85 & \\
\hline Amapola & 71.39 & 51.7 & 36.2 & 27.5 & 16.4 & 10.5 & 8.16 & \\
\hline Palo de sangre & 48.50 & 37.6 & 26.8 & 20.3 & 15.4 & 11.2 & 8.91 & \\
\hline Jobo & 84.34 & 61.4 & 39.6 & 21.6 & 16.3 & 11.8 & 8.65 & \\
\hline Laurel & 52.80 & 39.7 & 27.2 & 21.8 & 16.7 & 10.4 & 8.08 & \\
\hline Pasa'ak & 84.25 & 60.5 & 34.9 & 21.3 & 15.3 & 11.1 & 7.53 & \\
\hline Maca blanca & 115.52 & 79.8 & 56.8 & 45.7 & 33.7 & 26.7 & 18.7 & 11.79 \\
\hline Jolmashté & 49.80 & 36.4 & 25.6 & 19.4 & 13.8 & 9.2 & 7.49 & \\
\hline Palo de agua & 52.08 & 39.4 & 27.6 & 22.2 & 16.7 & 10.4 & 8.69 & \\
\hline Guacibán & 52.89 & 37.5 & 25.8 & 19.8 & 14.7 & 10.1 & 8.69 & \\
\hline Cedrillo & 80.53 & 59.4 & 36.5 & 29.4 & 23.7 & 17.2 & 13.91 & 9.43 \\
\hline Popiste & 53.28 & 45.6 & 31.3 & 27.5 & 21.4 & 15.9 & 12.65 & 9.15 \\
\hline
\end{tabular}

Evaluación de la calidad del secado de las especies de densidad baja. Como se aprecia en la Tabla 8, el porcentaje de volumen de madera perdido por efecto del proceso de secado, varia de $1.46 \%$ a $3.38 \%$, siendo mayor en el jolmasthé, pasa'ak, jobo, sac-chacáh, cedrillo y laurel. En el resto de las especies indicadas en la tabla de referencia, el porcentaje de volumen perdido no llegó a 3\%, lo que se encuentra dentro del rango normalmente aceptado (hasta $5 \%$ ) y que representa un volumen poco significativo desde el punto de vista económico, deduciéndose que la secuela utilizada fue apropiada con base en la calidad del secado de la madera.

El desarrollo de defectos en la madera se evaluó de manera semejante al inicio y al final del proceso de secado, considerando las grietas, las torceduras, el arqueamiento, el acanalamiento y el encorvado. Los promedios de la variación de la deformación de cada uno de los defectos analizados al final del secado de la madera, se indican en la Tabla 9. Como se puede apreciar, el desarrollo de estos defectos por el proceso de secado fue nulo y no afectó el número de unidades de corte en cada tabla, que se conservó prácticamente sin variación después del secado, lo que indica que la calidad de la madera respecto a su clasificación inicial no se modificó, o sea que una tabla de primera. antes del secado, al conservar el mismo número de unidades de corte al final del secado, se clasifica en la misma categoría de primera. Por el contrario, si la tabla aumentara el número de unidades de corte, podría conservar la misma superficie útil, pero se clasificaría en una categoría inferior. Con base en el análisis de los defectos antes y después del secado y de las unidades de corte de las tablas, se infiere que la secuela utilizada se puede considerar apropiada al no afectar la calidad de la madera. 
Tabla 8. Volumen de madera aprovechable antes y después del secado de las especies de baja densidad

\begin{tabular}{||l|c|c|c||}
\hline \multicolumn{1}{|c|}{ ESPECIES } & $\begin{array}{c}\text { VOLUMEN } \\
\text { ANTES DEL } \\
\text { SECADO } \\
(\%)\end{array}$ & $\begin{array}{c}\text { VOLUMEN } \\
\text { DESPUÉS } \\
\text { DEL SECADO } \\
(\%)\end{array}$ & $\begin{array}{c}\text { PÉRDIDA DE } \\
\text { VOLUMEN } \\
(\%)\end{array}$ \\
\hline Palo de picho & 93.36 & 91.25 & 2.11 \\
Sac-chacáh & 92.45 & 89.26 & 3.19 \\
Masamorro & 89.13 & 87.28 & 1.85 \\
Caoba & 87.67 & 86.21 & 1.46 \\
Zopo & 92.63 & 90.15 & 2.48 \\
Amapola & 88.74 & 86.09 & 2.65 \\
Palo de sangre & 90.36 & 88.58 & 1.78 \\
Jobo & 92.65 & 89.41 & 3.24 \\
Laurel & 91.45 & 88.33 & 3.12 \\
Pasa'ak & 92.69 & 89.32 & 3.37 \\
Maca blanca & 88.69 & 86.37 & 2.32 \\
Jolmashté & 93.56 & 90.18 & 3.38 \\
Palo de agua & 91.47 & 89.64 & 1.83 \\
Guacibán & 89.58 & 87.35 & 2.23 \\
Cedrillo & 92.43 & 89.27 & 3.16 \\
Popiste & 91.56 & 89.67 & 1.89 \\
\hline \hline
\end{tabular}

Tabla 9. Porcentaje promedio de deformaciones de la madera de las especies de baja densidad

\begin{tabular}{|c|c|c|c|c|c|c|c|c|c|c|c|c|}
\hline \multirow{3}{*}{ ESPECIES } & \multicolumn{6}{|c|}{ ANTES DEL SECADO } & \multicolumn{6}{|c|}{ DESPUÉS DEL SECADO } \\
\hline & \multirow{2}{*}{ PUC } & $G$ & TO & $A C$ & AR & EN & \multirow{2}{*}{ PUC } & $\mathrm{G}$ & TO & $A C$ & $A R$ & EN \\
\hline & & $(\%)$ & $(\%)$ & $(\%)$ & (\%) & $(\%)$ & & (\%) & $(\%)$ & $(\%)$ & $(\%)$ & (\%) \\
\hline & 2.54 & 0.00 & 0.00 & 0.00 & 0.00 & 0.00 & 2.86 & 0.00 & 0.00 & 0.00 & 0.00 & 0.00 \\
\hline & 3.23 & 0.00 & 0.00 & 0.00 & 0.00 & 0.00 & 3.65 & 0.00 & 0.00 & 0.00 & 0.00 & 0.00 \\
\hline & 2.28 & 0.00 & 0.00 & 0.00 & 0.00 & 0.00 & 2.47 & 0.00 & 0.00 & 0.00 & 0.00 & 0.00 \\
\hline & 2.63 & 0.00 & 0.00 & 0.00 & 0.00 & 0.00 & 2.94 & 0.00 & 0.00 & 0.00 & 0.00 & 0.00 \\
\hline & 2.75 & 0.00 & 0.00 & 0.00 & 0.00 & 0.00 & 3.04 & 0.00 & 0.00 & 0.00 & 0.00 & 0.00 \\
\hline l & 2.49 & 0.00 & 0.00 & 0.00 & 0.00 & 0.00 & 2.83 & 0.00 & 0.00 & 0.00 & 0.00 & 0.00 \\
\hline ngre & 2.37 & 0.00 & 0.00 & 0.00 & 0.00 & 0.00 & 2.56 & 0.00 & 0.00 & 0.00 & 0.00 & 0.00 \\
\hline & 3.35 & 0.00 & 0.00 & 0.00 & 0.00 & 0.00 & 3.42 & 0.00 & 0.00 & 0.00 & 0.00 & 0.00 \\
\hline & 2.86 & 0.00 & 0.00 & 0.00 & 0.00 & 0.00 & 3.06 & 0.00 & 0.00 & 0.00 & 0.00 & 0.00 \\
\hline & 3.12 & 0.00 & 0.00 & 0.00 & 0.00 & 0.00 & 3.47 & 0.00 & 0.00 & 0.00 & 0.00 & 0.00 \\
\hline & 2.92 & 0.00 & 0.00 & 0.00 & 0.00 & 0.00 & 3.25 & 0.00 & 0.00 & 0.00 & 0.00 & 0.00 \\
\hline & 2.54 & 0.00 & 0.00 & 0.00 & 0.00 & 0.00 & 2.72 & 0.00 & 0.00 & 0.00 & 0.00 & 0.00 \\
\hline & 2.82 & 0.00 & 0.00 & 0.00 & 0.00 & 0.00 & 3.96 & 0.00 & 0.00 & 0.00 & 0.00 & 0.00 \\
\hline & 2.76 & 0.00 & 0.00 & 0.00 & 0.00 & 0.00 & 2.93 & 0.00 & 0.00 & 0.00 & 0.00 & 0.00 \\
\hline & & 0.00 & 0.00 & 0.00 & 0.00 & 0.00 & 3.42 & 0.00 & 0.00 & 0.00 & 0.00 & 0.00 \\
\hline & 2.43 & 0.00 & 0.00 & 0.00 & 0.00 & 0.00 & 2.65 & 0.00 & 0.00 & 0.00 & 0.00 & 0.00 \\
\hline
\end{tabular}

PUC = promedio de Unidades de Corte

$\mathrm{G}=$ grietas

$\mathrm{TO}=$ torceduras

$A C=$ acanalamiento

$A R=$ arqueamiento

$\mathrm{EN}$ = encorvamiento 
Evaluación de la pérdida de humedad durante el secado de las especies de alta densidad. Para las especies con densidad básica mayor a $0.60 \mathrm{gr} / \mathrm{cm}^{3}$, de la evaluación de las tablas, antes y después del proceso, se considera que la secuela (Tabla 6) fue favorable al no desarrollarse defectos por el secado que afectaran la calidad de la madera.

Con base en el tiempo de secado requerido de las diferentes especies estudiadas, es factible diferenciar 4 grupos: el primero integrado por granadillo, ya'axnik y guaité, con un contenido de humedad inicial y final muy similar, alrededor de $30 \%$ y $7 \%$ respectivamente, requiriendo un tiempo promedio de secado de 10 días (Tabla 10). El segundo grupo estaría integrado por pimientillo, canshán, tinco y chicozapote, con fluctuaciones de contenido de humedad inicial de $22.95 \%$ en canshán hasta un $56.58 \%$ en tinco. En este grupo no se observa una tendencia regular en la pérdida de humedad, puesto que la de menor $\mathrm{CH}$ inicial no necesariamente se secó más rápido. La madera de tinco con mayor $\mathrm{CH}$ inicial en relación a las otras tres, después de 14 días de secado presentó un contenido de humedad similar a canshán y chicozapote que inicialmente tenían un $\mathrm{CH}$ menor. Otro grupo podría estar integrado por trementino, machiche y corazón azul, también con $\mathrm{CH}$ iniciales diferentes y después de 22 días de secado, muy similares y en un promedio de $8 \%$. El cuarto grupo estaría integrado por chacahuanté, bayo, luín, chiquinib, ramón, guapaque y chakté, también con un contenido de humedad inicial muy variado y con un periodo de secado promedio de 25 días para un $\mathrm{CH}$ de alrededor del 8\% (Tabla 10).

Considerando los tiempos o periodos de duración del proceso de secado y el comportamiento de la madera de las especies estudiadas, la integración de los cuatro grupos indicados es la más adecuada. Sin embargo, desde el punto de vista práctico, para realizar el proceso de secado en estufa, seguramente lo más recomendable sería integrar únicamente dos grupos, el primero formado por guaité, granadillo, pimientillo, canshán, tinco y chicozapote mientras que el segundo grupo estaría formado por trementino, bayo, luín, chiquinib, ramón, guapaque, chacahuanté chakté y corazón azul. Posiblemente el primer grupo se podría secar con otra secuela más rápida para reducir el tiempo de secado y al segundo grupo se le aplicaría la misma secuela analizada en este trabajo.

\section{Evaluación de la calidad del secado de} las especies de densidad alta. El porcentaje de volumen de madera perdido por efecto del proceso de secado, varía de $2.16 \%$ a $3.14 \%$ (Tabla11), y únicamente cuatro especies rebasaron $3 \%$, el canshán, el granadillo, el guaité y el chicozapote. En las otras 11 especies, el porcentaje de volumen perdido no llegó a 3\%, lo cual representa un volumen poco significativo desde el punto de vista económico, por lo que la secuela utilizada se puede considerar apropiada con base en la calidad del secado de la madera.

Respecto a los defectos o degradación de la madera por el secado, (grietas, torceduras, arqueamiento, acanalamiento, encorvado), se evaluaron al inicio y al final del proceso, determinándose un efecto insignificante en el número de Unidades de Corte, que prácticamente se mantuvieron sin variación antes y después del secado, lo que indica que no se afectó la calidad de la madera (Tabla 12).

Los defectos analizados antes y después del secado (grietas, torceduras, acanalamiento, arqueamiento y encorvado), no afectaron la calidad de la madera porque la secuela utilizada fue moderada. La secuela que se aplicó asegura un buen secado de las 17 especies estudiadas, pero se considera que se podría acelerar aplicando condiciones más severas al grupo de especies integrado por guaité, granadillo, pimientillo, canshán, tinco y chicozapote. Las otras 11 especies, como 
Tabla 10. Pérdida de humedad durante el secado en las maderas de alta densidad

\begin{tabular}{|c|c|c|c|c|c|c|c|c|c|c|c|c|}
\hline \multirow{3}{*}{ ESPECIES } & \multicolumn{12}{|c|}{ TIEMPO DE SECADO EN DÍAS } \\
\hline & 0 & 2 & 4 & 6 & 8 & 10 & 12 & 14 & 16 & 18 & 22 & 25 \\
\hline & $(\%) \mathrm{CH}$ & $(\%) \mathrm{CH}$ & $(\%) \mathrm{CH}$ & $(\%) \mathrm{CH}$ & $(\%) \mathrm{CH}$ & $(\%) \mathrm{CH}$ & $(\%) \mathrm{CH}$ & $(\%) \mathrm{CH}$ & $(\%) \mathrm{CH}$ & $(\%) \mathrm{CH}$ & $(\%) \mathrm{CH}$ & (\%)CH \\
\hline Pimientillo & 36.53 & 32.35 & 28.38 & 22.12 & 17.76 & 13.97 & 11.23 & 8.52 & & & & \\
\hline Treme & 42.66 & 37.33 & 29.39 & 23.72 & 18.21 & 14.43 & 12.68 & 10.25 & 9.72 & 9.51 & 8.15 & \\
\hline Chacahunté & 40.35 & 35.15 & 32.87 & 27.64 & 21.92 & 16.57 & 13.86 & 11.89 & 11.55 & 11.11 & 9.06 & 8.12 \\
\hline Canshán & 22.95 & 21.44 & 17.22 & 15.18 & 13.70 & 11.53 & 10.19 & 8.20 & & & & \\
\hline & 56.58 & 46.19 & 33.76 & 25.23 & 18.10 & 13.5 & 11.33 & 8.80 & & & & \\
\hline & 32.02 & 26.88 & 20.69 & 16.16 & 0.64 & 8.60 & & & & & & \\
\hline Ya'axnik & 26.07 & 23.40 & 16.20 & 11.49 & 9.45 & 7.85 & & & & & & \\
\hline Bayo & 51.39 & 45.09 & 37.82 & 32.88 & 27.59 & 23.62 & 21.56 & 18.33 & 17.25 & 17.12 & 11.5 & 9.65 \\
\hline Luín & 27.98 & 25.39 & 21.43 & 19.34 & 17.70 & 15.28 & 13.97 & 11.95 & 11.11 & 10.97 & 9.01 & 8.12 \\
\hline quinib & 62.16 & 57.79 & 52.04 & 47.92 & 42.71 & 38.66 & 35.22 & 30.21 & 26.64 & 24.92 & 12.67 & 9.93 \\
\hline & 32.35 & 29.14 & 23.90 & 21.04 & 18.42 & 15.54 & 13.90 & 11.46 & 10.73 & 10.63 & 9.02 & 8.56 \\
\hline & 33.45 & 30.68 & 26.55 & 24.1 & 22.26 & 19.97 & 18.63 & 16.20 & 15.49 & 15.4 & 7.87 & \\
\hline & 43.03 & 39.59 & 32.85 & 29.33 & 26.87 & 23.74 & 22.21 & 19.60 & 18.95 & 17.8 & 9.86 & 8.60 \\
\hline & 30.50 & 25.65 & 19.96 & 15.87 & 10.94 & 7.86 & & & & & & \\
\hline & 26.16 & 24.18 & 20.71 & 18.82 & 17.27 & 15.04 & 13.68 & 12.70 & 11.26 & 10.82 & 9.05 & 7.90 \\
\hline zón azul & 25.01 & 22.75 & 18.61 & 17.06 & 15.83 & 13.46 & 11.82 & 9.70 & 9.29 & 9.1 & 8.10 & \\
\hline Chicozapote & 26.18 & 23.53 & 20.14 & 17.85 & 15.02 & 12.44 & 10.51 & 8.20 & & & & \\
\hline
\end{tabular}

Tabla 11. Volumen de madera aprovechable de especies de alta densidad, antes y después del secado

\begin{tabular}{||l|c|c|c||}
\hline \multicolumn{1}{|c|}{ ESPECIES } & $\begin{array}{c}\text { VOLUMEN } \\
\text { ANTES DEL } \\
\text { SECADO } \\
(\%)\end{array}$ & $\begin{array}{c}\text { VOLUMEN } \\
\text { DESPUÉS } \\
\text { DEL SECADO } \\
(\%)\end{array}$ & $\begin{array}{c}\text { PÉRDIDA DE } \\
\text { VOLUMEN }\end{array}$ \\
\hline Pimientillo & 85.47 & 82.74 & 2.73 \\
Trementino & 89.26 & 86.68 & 2.58 \\
Chacahuanté & 87.34 & 85.18 & 2.16 \\
Canshán & 82.76 & 79.64 & 3.12 \\
Tinco & 81.43 & 78.48 & 2.95 \\
Granadillo & 83.52 & 80.38 & 3.14 \\
Ya'axnik & 82.38 & 79.42 & 2.96 \\
Bayo & 88.54 & 86.09 & 2.45 \\
Luín & 84.48 & 82.11 & 2.37 \\
Chiquinib & 83.74 & 81.58 & 2.16 \\
Ramón & 85.53 & 83.36 & 2.17 \\
Machiche & 84.38 & 81.82 & 2.56 \\
Guapaque & 87.47 & 84.72 & 2.75 \\
Guaité & 79.84 & 76.73 & 3.11 \\
Chakté & 82.96 & 80.21 & 2.75 \\
Corazón azul & 86.15 & 83.36 & 2.79 \\
Chicozapote & 88.36 & 85.23 & 3.13 \\
\hline \hline
\end{tabular}


Tabla 12. Evaluación de los defectos de las maderas de alta densidad, antes y después del secado

\begin{tabular}{|c|c|c|c|c|c|c|c|c|c|c|c|c|}
\hline \multirow{3}{*}{ ESPECIES } & \multicolumn{6}{|c|}{ ANTES DEL SECADO } & \multicolumn{6}{|c|}{ DESPUÉS DEL SECADO } \\
\hline & \multirow{2}{*}{ PUC } & $\mathrm{G}$ & TO & $A C$ & AR & EN & \multirow{2}{*}{ PUC } & G & TO & $A C$ & $\mathrm{AR}$ & EN \\
\hline & & $(\%)$ & $(\%)$ & $(\%)$ & (\%) & $(\%)$ & & (\%) & (\%) & (\%) & $(\%)$ & (\%) \\
\hline Pimientillo & 2.35 & 0.00 & 0.00 & 0.00 & 0.05 & 0.08 & 2.63 & 0.61 & 0.03 & 0.58 & 0.31 & 0.32 \\
\hline Trementino & 2.62 & 0.00 & 0.00 & 0.00 & 0.00 & 0.12 & 3.85 & 1.36 & 0.06 & 0.09 & 0.08 & 0.45 \\
\hline Chacahuanté & 2.75 & 0.00 & 0.00 & 0.03 & 0.11 & 0.16 & 2.97 & 1.47 & 0.12 & 0.76 & 0.59 & 0.48 \\
\hline Canshán & 3.12 & 0.00 & 0.00 & 0.00 & 0.03 & 0.09 & 3.89 & 0.63 & 0.04 & 0.67 & 0.29 & 0.23 \\
\hline Tinco & 2.75 & 0.00 & 0.00 & 0.00 & 0.07 & 0.10 & 2.98 & 0.39 & 0.06 & 0.42 & 0.18 & 0.36 \\
\hline Granadillo & 3.12 & 0.00 & 0.00 & 0.00 & 0.08 & 0.08 & 3.98 & 0.26 & 0.03 & 0.53 & 0.29 & 0.37 \\
\hline Ya'axnik & 2.29 & 0.00 & 0.00 & 0.05 & 0.07 & 0.13 & 2.37 & 0.59 & 0.12 & 0.48 & 0.31 & 0.42 \\
\hline Bayo & 2.67 & 0.00 & 0.00 & 0.03 & 0.06 & 0.19 & 2.96 & 1.54 & 0.14 & 0.68 & 0.22 & 0.69 \\
\hline Luín & 2.73 & 0.00 & 0.00 & 0.00 & 0.12 & 0.18 & 2.98 & 1.72 & 0.17 & 0.17 & 0.35 & 0.71 \\
\hline Chiquinib & 3.14 & 0.00 & 0.00 & 0.02 & 0.09 & 0.21 & 3.35 & 2.12 & 0.28 & 0.49 & 0.43 & 0.57 \\
\hline & 2.37 & 0.00 & 0.00 & 0.00 & 0.04 & 0.15 & 2.45 & 1.23 & 0.07 & 0.25 & 0.37 & 0.64 \\
\hline iche & 2.14 & 0.00 & 0.00 & 0.00 & 0.11 & 0.17 & 2.56 & 1.86 & 0.08 & 0.29 & 0.59 & 0.69 \\
\hline que & 3.12 & 0.00 & 0.00 & 0.00 & 0.09 & 0.16 & 3.37 & 2.16 & 0.13 & 0.17 & 0.27 & 0.32 \\
\hline & 2.63 & 0.00 & 0.00 & 0.00 & 0.00 & 0.08 & 3.74 & 0.37 & 0.04 & 0.45 & 0.09 & 0.19 \\
\hline & 3.11 & 0.00 & 0.00 & 0.03 & 0.15 & 0.18 & 3.46 & 1.28 & 0.22 & 0.86 & 0.62 & 0.53 \\
\hline azul & 2.43 & 0.00 & 0.00 & 0.00 & 0.12 & 0.14 & 2.89 & 2.35 & 0.14 & 0.27 & 0.45 & 0.47 \\
\hline Chicozapote & 3.18 & 0.00 & 0.00 & 0.00 & 0.06 & 0.09 & 3.98 & 0.47 & 0.05 & 0.35 & 0.26 & 0.25 \\
\hline
\end{tabular}

se señaló anteriormente, se pueden secar con la secuela analizada en este estudio.

\section{CONCLUSIONES Y RECOMENDACIONES}

Se considera que las secuelas de secado aplicadas a los dos grupos de especies tropicales que se integraron con base en su densidad, fueron apropiadas por los resultados generados en cuanto a la calidad de la madera, prácticamente no hubo cambio en el volumen aprovechable de la madera, antes y después del secado, ni tampoco hubo cambios en el número de unidades de corte, por lo que se concluye que el proceso de secado con las secuelas utilizadas no afectò la calidad de la madera.
En el grupo de especies de densidad baja y media, es posible integrar un subgrupo de 10 especies que se podrían secar con una secuela más rápida que la indicada en la Tabla 5, con la finalidad de reducir el tiempo de secado y optimizar el uso de las estufas con ciclos más cortos de secado.

Para el caso de las especies de densidad alta, 7 especies se podrían secar con una secuela más severa que la señalada en la Tabla 6 , con la finalidad de reducir el tiempo de secado e incrementar la capacidad de producción de las estufas.

De no ser posible subagrupar a las especies dentro de cada categoría de duras y blandas, para aplicarles una secuela más rápida a las especies con mayor velocidad de secado, se sugiere que el contenido de 
humedad y dimensiones de la madera sean similares en cada grupo, para evitar discrepancias acentuadas en los tiempos de secado.

Debido a que las dos secuelas analizadas se evaluaron únicamente con una carga de madera y no fue posible realizar ninguna repetición del proceso de secado, se sugiere que las secuelas analizadas en este estudio se apliquen con una supervisión continua del proceso, evaluando diariamente su desarrollo a través de la calidad del secado de las maderas de los dos grupos de especies señalados.

\section{REFERENCIAS}

Bello, E.D. 1966. The seasoning characteristics of some Philippine woods. Wood Preservation Division Rep. 1(6).

Boone, R. S., J.C. Kozlik, J. P. Bois y M.E. Wengert. 1988. Dry kiln schedules for commercial woods, temperate and tropicals. USDA. Forest Service. FPLGTR-57. 158 p.

Bramhall, G. 1975. Calculating kiln schedule changes. Canadian Forest Industries. September Issue. 3 p.

Bramhall, G. y R.W. Wellwood. 1976. Kiln drying of western canadian lumber. WFPL VP-X-159. $112 \mathrm{p}$.

Casin, R.F., M.G. Laxamana y G.Y. Tamayo. 1980. Kiln-drying schedules of some Philippine commercial wood species. The Philippine Lumberman. 26(3):14-26.

Dirección de Desarrollo Industrial (DDI), 1991. Proyecto de Norma de Clasificación de Madera Aserrada de Latifoliadas. Subsecretaría Forestal. SARH. México. $36 \mathrm{p}$.
Dirección General de Normas (DGN), NOMEE-95-1980. Emvase y Embalaje.Madera.- Determinación de los defectos en maderas aserradas $y$ cepilladas. Secretaría de Patrimonio y Fomento Industrial. México. 20 p.

Keenan, F.J. y M. Tejeda. 1984. Tropical timber for building materials in the Andean Group countries of South America.IDRC-TS49e. Intern. Devel. Research Center. Ottawa, Canada

Ma, T.P. 1972. Mixed hardwood kiln drying experiment-kiln drying of hardwood grown in northern part of Taiwan. Co-op. Bull. 2. Taiwan Forest Research Institute Taipei.

Ma, T.P. 1972. Mixed hardwood kiln drying experiment-kiln drying of hardwood grown in the central part of Taiwan. Co-op. Bull. 3. Taiwan Forest Research Institute. Taipei.

Hildebrand, R. 1970. Kiln drying of sawn timber. Alemania. 199 p.

Pratt, H.G. 1974. Timber drying manual. Princess Risborough Lab. Inglaterra. $152 \mathrm{p}$.

Rasmussen, E.F. 1968. Dry kiln-Operator's manual. FPL. Forest Service, USDA . Agriculture Handbook No.188. Madison, WI. EUA. 197 p.

Simpson, T.W. 1991. Dry kiln operator's manual. USDA. For. Serv. FPL. Agriculture Handbook. No. 188. Madison, WI. EUA. 274 p.

Simpson, T.W. y K.C. Baah. 1989. Grouping tropical wood species for kiln drying. USDA. Forest Service. FPLRN-0256. Madison, WI. EUA. 14 p.

Torelli, N. 1983. Estudio promocional de 43 especies forestales tropicales mexicanas. SFF. SARH. México. 67 p. 